\title{
Investigation on Chinese Learning Methods for Primary School Students of Rural Area
}

\author{
Zheng Zhang ${ }^{\mathrm{a}}$, Jun Yang ${ }^{\mathrm{b}}$ \\ School of Education and Sports, Bohai University, Jinzhou, 121013, China \\ a147994621@qq.com, byj690213@126.com
}

Keywords: rural area; primary school students; Chinese learning methods; investigation

\begin{abstract}
Chinese curriculum not only directly affects the formation of students' language literacy, but also affects the students' learning of other courses. Chinese learning methods in rural primary school students directly affect the effectiveness of their language learning, influence the formation of their language literacy, which ultimately affect student learning other courses. In this paper, Chinese learning method in rural primary school students as the research object, through the investigation on the mastery of the Chinese learning principle, Chinese learning strategies and general learning method and the specific Chinese learning methods, to analyze their current situation to master the Chinese learning method, proposed the preliminary improvement strategies, both to help students master the basic principles of learning, learning strategies and general learning methods, to help students master the specific Chinese learning method.
\end{abstract}

\section{Introduction}

Language is mankind's most important communication tool and information carrier, is an important part of human culture. The unity of instrumentality and humanistic is the basic characteristics of Chinese courses. The tool characteristic of Chinese discipline decides that it is the base of studying other subjects, and the humanities character of Chinese subject decides that it has a comprehensive and profound influence on the students' outlook on life, world outlook and values. Thus, the Chinese is called the foundation of basic education. Rural education has always been a weak zone of education in China [1]. Due to the different family background, growing environment, teachers, school software and hardware, as well as the surrounding social environment, rural students and urban students in Chinese learning there is a natural difference. This natural differences lead to rural students' Chinese learning levels compared with urban students there is a huge gap [2]. Research on the particularity of rural students' Chinese learning is conducive to narrowing the gap. Learning sciences believes that any study is a positive and active learning internalization process. And whether learners have the willingness to take the initiative to learn, whether to master the method of autonomous learning is particularly important [3]. In this paper, the Chinese learning methods of rural primary school students as the research object, the initial exploration of rural primary school students Chinese e learning problems, and the initial improvement of rural primary school students Chinese learning strategies.

\section{The Definition of Chinese Learning Methods}

Academic systematically discusses Chinese learning method is very few. In this paper, based on a vast amount of reference material, citing Mr. Wang Guanglong classification of Chinese learning methods: Chinese learning method as a discipline of learning method, it contains four levels of learning methods, namely Chinese learning principles and Chinese learning strategies levels; applicable general learning method level of Chinese discipline learning; Chinese subject specific learning method level; Chinese learning specific learning method level [4]. According to the above classification, the author for the Chinese learning principles in four levels of Chinese learning methods, Chinese learning strategies and general learning methods to examine together, the Chinese subjects specific learning methods and Chinese learning specific learning method to examine together, and Chinese learning content that is literacy and writing word, reading, writing, oral 
communication as the clue, two rural primary schools in Jinzhou and a rural primary school in Benxi, a total of 296 rural primary school students to master the Chinese learning method were investigated.

\section{Investigation on the Chinese Learning Principle, Chinese Learning Strategies and General Learning Method}

We designed the "When you find you're absent-minded can come back in time to learning again?" And "Can you keep up with the teacher thinking and positive thinking problems when the teacher is teaching?" "Every day, every week you have some learning plan?" "Can you weekly, monthly, per semester of Chinese knowledge summarized, and summed it?" "Do you have the habit of preparing lessons before class, listening carefully in class, and reviewing after class?" "Can you intend to do some exercises to consolidate own learning effect?" the several problems related to the problems of learning principles and general learning method, the enthusiasm of students to answer (can, yes, you can) in 32\%, the rest of answer was relatively positive, negativity answer and so on. Visible, the majority of rural primary school students to master learning principles and general learning methods is ideal, learning enthusiasm is not high.

\section{Investigation on Literacy and Writing Learning Method}

Here the design of the "learning Chinese Pinyin, how do you remember the initials and finals?" "Encountered do not know the word, you will usually how to do" "When remember a word shape, your approach is?" "How do you to write a good hand?" "When practice writing, if you carefully observe the frame structure between a word?" and other issues, the answer set is basically follow the negative answer, relatively positive answer, positive answer three levels. This group of problems, the positive answer of students accounted for $35.6 \%-69.9 \%$, indicating that there is $1 / 3$ to $7 / 10$ of the students mastered the more appropriate of the read and write method; but negativity answer (rote, leave it in there and don't manage it, a stroke memory, the teacher guidance, have never noticed) also accounted for a considerable proportion, respectively were $21.0 \%, 10.9 \%$, $25.2 \%, 54.4 \%, 18.2 \%$. This shows that there is a considerable number of primary school students do not learn to high school students should have the ability of the literacy and write method, still use the most original, the most awkward method to learn, which will greatly affect their Chinese learning achievement.

\section{Investigation on Reading Method}

Several problems here concerning reading: "Can you take a question to read the article?" "Can imagine scene while you read the book?" "Do you have a habit to mark?" "What do you understand the reading aloud?" "How do you usually recite the text?" "When you do reading notes, what do you use method?" for the first three questions that negativity answer of students (never like this, cannot think, no designated circle habits), respectively $10.0 \%, 10.3 \%, 22.5 \%$, indicating a $10.0 \%$ $-22.5 \%$ of the students do not develop basic reading methods and habits; relative positive answer (sometimes do, sometimes can, teachers stressed punctuate) accounted for the total of $61.1 \%, 47.7 \%$, $36.2 \%$, reading habits and methods to develop this part of the students is not yet stable, it needs to be strengthened. The fourth issue relates to reading understanding, on this issue, biased, incomplete understanding accounted for 38\%, indicating that many students will not be very good for reading aloud; fifth question relates to recite method, wherein the positive answer "to find some keywords recite" accounted for $17.3 \%$. It can be seen that only a few students can find the key words in the article as a clue to the memory. The sixth question relates to a method of reading notes, "make a mark in the book" accounted for 18.2\%, "excerpts wonderful sentence and words" accounted for $52.9 \%$, "write the book review" accounted for $28.9 \%$, the majority of students used to reading notes of marks and excerpts from the primary reading notes method, and the need for a strong 
generalization ability and appreciation ability, the reading notes method in the form of book review not many students to grasp.

\section{Investigation on Writing Method}

The first three issues which were part of the survey relates to the application of the accumulation of similar words, Imitative ability, expressions way, which do not have any of these able students accounted for $30.3 \%, 26.4 \%$, 26.4\%, indicating that the part of the students for writing lack of basic the accumulation of consciousness and ability; and with the basic awareness and ability of students accounted for respectively $40.2 \%, 46.5 \%, 46.5 \%$, this part of the students' awareness is not clear enough, ability is not stable enough, and needs teacher actively guide and strengthening; and fully grasp the ability of these students are less than 30\%, indicating that students master the writing skills of students is less than $1 / 3$. The fourth and the fifth issue involves the student's initiative to amend and studying the teacher's ideas the two aspects, including "never modify" and "seldom read" accounted for $28.2 \%$, 24.9\%, this part of the students did not modify consciousness; and "sometimes modify" and "basic can read teacher comments" accounted for 42.3\%, 33.4\%, this part of the students by the teacher stressed and guide is can develop good modify consciousness. The last question is the habit of practicing writing diary, which "never written" accounted for 23.1\%, "There is some thoughts to write" accounted for 54.4\%, "Every day I write a diary" accounted for $22.5 \%$, showing that the diary practicing writing, the most important method of writing, students master is not very good, there are still nearly a quarter of the students in addition to class composition simply never write a paragraph of text, this situation is very difficult to write well.

\section{Investigation on Oral Communication Method}

This group of content involves the "development of the content of speech, willing to speak in public awareness and ability, to speak the idea is clear or not, obedient etiquette capabilities" four aspects. For these four questions, respectively make negative answer (not, never spoke) were accounted for $16.4 \%, 28 \%, 20.9 \%, 20.3 \%$, indicating that nearly one-fifth of the students do not have to take the initiative with others oral communication awareness and ability, it would seriously affect the development of their language abilities; and for four issues to make this relatively positive answer (basically) respectively accounted for 56.5\%, 49.2\%, 50.2\%, 35.3\%, showing a nearly half of the students initially with oral communication awareness and ability; the positive answer of four questions (so often) accounted for only $27.1 \%, 22.8 \%, 28.9 \%, 44.4 \%$, visible, able to skillfully use oral communication skills not a lot of students, the basic $30 \%$ or less (the last one exception). From the analysis results, the ability of oral communication in today's society is very important, there are still many needs to improve and upgrade the place for rural primary school students.

\section{Upgrade Chinese Learning Method Strategies of Rural Primary School Students}

(1) To enable students to master the basic principles of learning, learning strategies and general learning method. Chinese as a basis discipline, it has some general learning principles with other disciplines, learning strategies and general learning method. This requires teachers in the usual teaching intentional penetration to students, and constantly in the future of teaching to lead students to use repeatedly, and ultimately achieve proficiency applications. There are many of these basic learning principles and learning methods, such as consciously relatively similar, confusing knowledge, consciously broaden their knowledge, increase more information, more in mind, to accumulate basic knowledge of the discipline, each stage has a certain learning programs and a clear sense of purpose, can concentrate energy listening and learning to think, to develop the habit of previewing and reviewing effective, develop the habit of timely summarized, keep active exercises to consolidate the learned habits, to develop experience do not understand the contents with the help of reference books, contact the context guess, seek the help of others and other habits. 
In fact, these strategies and methods, that is, educational psychology student said cognitive strategies, metacognitive strategies, resource management strategy [5]. Master these strategies and methods, not simply relying on the explanation of method and strategy, can be reached, and the need to combine appropriate examples in the teaching, to vivid and effective form to teach students, students can grasp good, and constantly migrated to the future study and other subjects learning [6].

(2) To enable students to master the specific literacy, writing methods. Depending on the content of Chinese learning, on learning Chinese pinyin, should form loved to equip students master the pinyin tool in a relaxed atmosphere, you can play games, storytelling and other ways for students to learn and memory; the memory shape, try to contact the radicals and learned words, by adding a plus, minus one minus, for a change in the method of linking the old and new knowledge, so that comparison of memory is conducive to expanding literacy, writing aspects may guide students to carefully observe the words between frame structure, the proportion of each part, so that students copy copybook, practice constantly compare the way gradually write beautiful calligraphy [7]. Of course, this put forward higher requirement for writing ability of teachers, and teachers should not only teach students to write, but also to teach students in a proper way to master the method of writing Chinese characters.

(3) Enable students to master the proper reading method. Chinese proficiency in reading ability is a very important content, and its level will directly determine a person's level of language literacy. So, how to improve students' reading method? Can be combined with the characteristics of Chinese teaching in primary school, teach students some method, with problems to read, while reading thinking best can reproduce the scene in the book, make a mark in order to highlight the key, master circle a mark, excerpts, write book review, and a variety of method of reading notes. [8] But also allows students to grasp the fluent and full of feelings of reading methods, to keywords for clues rapid recitation methods and so on. Master these methods in favor of the rapid increase in students' reading skills.

(4) To enable students to master the basic writing method. Writing is mainly the training of specific writing methods and the cultivation of interest in writing. Including the accumulation of necessary phrases, a certain amount of imitative writing practice, three expressions ways of repeated training and proficient, good writing habits, as well as enable students to develop the habit of keeping a diary of everyone. Diary is the most effective form of daily practicing writing, keeping a diary is not only able to exercise their own sense of language, and can exercise their own thinking, but also cultivate their own temperament. With good interest and appropriate methods, students will be able to love writing, good at writing [9].

(5) To enable students to understand the specific oral communication methods. Oral communication is verbal language communication, it occupy an increasingly important position in real life. But by the influence of traditional culture "quick in action and less talk," our oral communications have not been able to get the attention it deserves. Students from an early age how to get a good grasp of oral communication methods? First is to develop the courage to speak in public, willing to speak in public, be able to seize the opportunity in front of people talking about, and secondly to train students' ability of oral communication. Such as constantly enrich the ability of the content of the conversation, to constantly will read books and newspapers, listening to the radio, heard the content timely to enrich their conversation. Another example is the ability to quickly sort out the idea of talking, can according to certain logical order, with certain conjunctions will speak the contents of a coherent expression. Finally, let students to develop good oral communication etiquette, such as a good listener, timely response and attention, appropriate lead to new topics and so on. [10] So that students early love to communicate with people, good at communicating with people.

\section{Conclusion}

Of course, improving primary school students' Chinese learning method is not overnight things, teachers need to pay the arduous efforts in teaching can be achieved initial results; a variety of 
learning methods is not completely separated, and even many of the method itself is mutual accommodation and mutual promotion; again, improve rural primary school students' Chinese learning method, but also for rural students basis poor, knowledge widowed, but rich experience of rural life the features have targeted, both all the general teaching guide, individualized individual guidance. In this way, we can effectively improve the rural primary school students' Chinese learning methods, so as to enhance their language literacy.

\section{Acknowledgement}

This work is supported by social science fund project of Liaoning province (L13CZZ044): Phenomenon analysis and countermeasure research on 'vulgarization' for college students' life world; A-class teaching reform project of Bohai university in 2014: Path study on normal students in teachers' professional ability training on the background of state test of teachers' qualification; 2014 annual "A" class teaching reform project in education department of Liaoning province (UPRP20140249).

\section{References}

[1] DOC88, "Ministry of education of the people's Republic of China: Full time compulsory $\begin{array}{lllll}\text { education } \quad \text { Chinese } \quad \text { curriculum } & \text { standard }\end{array}$ http://baike.baidu.com/link?url=VEq1p3ZLoerQrmbdPZSI-Yk5b1-CaptupJ8mt7AhLY1ePHV Vz2mpiq16N-CHVd3z6z5Jp8RYO55MyoZfanLreWaZMr8XfhMtAbWC90hD-sW, 2015-7-20.

[2] L. Zhao, "Predicament and outlet: Path exploration of urban and rural educational equity realization," Education Exploration, vol. 22, no. 6, pp. 55-58, 2014.

[3] Research group of teachers' qualification examination proposition, "Educational psychology," Central China normal university press, pp. 167, 2012.

[4] G. L. Wang, Theory of Chinese learning methods," Journal of Shanxi normal university (social science edition), vol. 24, no. 7, pp.133-137, 2003.

[5] Y. S. Chang, "Educational psychology," Liaoning normal university education publishing, pp. 116-117, 2010.

[6] Y. Luo, Z. T. Chen, "Education," Liaoning normal university education publishing, pp. 30-31, 2005.

[7] Z. H. Wu. Chen, "Primary school Chinese curriculum and teaching theory," Beijing normal university press, pp. 237-238, 2004.

[8] J. Yang, "The primary school Chinese new curriculum teaching theory," World books publishing house, pp. 105-106, 2012.

[9] Recent blog, "Primary school Chinese composition teaching methods," http://blog.sina.com.cn/s/blog_6fff6d7d0101ehgz.html, 2016-2-25.

[10] P. Jiang, "Primary school Chinese curriculum and teaching (version 2)," Higher education press, pp. 189, 2004. 